Fernanda Monteiro de Paula Siqueira ${ }^{1}$

Cezar Alencar de Lima Rezende ${ }^{2}$

Alexandre de AlmeidA BarRa ${ }^{3}$

Artigos originais

Palavras-chave

Neoplasias mamárias/diagnóstico Neoplasias mamárias/ultra-sonografia Neoplasias mamárias/patologia

Exame físico

Ultra-sonografia mamária

Mamografia

Prognóstico

Keywords

Breast neoplasms/diagnosis Breast neoplams/ultrasonography

Breast neoplasms/pathology

Physical examination

Ultrasonography, mammary

Mammography

Prognosis

\section{Correlação entre o exame clínico, a mamografia e a ultra-sonografia com o exame anatomopatológico na determinação do tamanho tumoral no câncer de mama}

\author{
Correlation between clinical examination, mammography and \\ ultrasonography with histopathological exam in the determination \\ of tumor size in breast cancer
}

\section{Resumo}

OBJETIVO: avaliar qual o melhor método entre o exame clínico (EC), a mamografia (MMG) e a ultra-sonografia (US) na determinação pré-operatória do tamanho tumoral no câncer de mama, tendo como referência o exame anatomopatológico. MÉTODOS: foram incluídas neste estudo 184 pacientes com lesões mamárias detectadas por MMG e US, palpáveis ou não, e que foram submetidas a ressecção cirúrgica do tumor, com diagnóstico histopatológico de câncer de mama. O maior diâmetro tumoral foi avaliado por EC, MMG e US por um mesmo examinador, e a medida obtida por cada método foi correlacionada com o diâmetro máximo obtido pelo exame anatomopatológico. A análise comparativa foi feita por meio do coeficiente de correlação de Pearson (r). RESULTADOS: o coeficiente de correlação de Pearson encontrado entre o exame anatomopatológico e o EC foi 0,8; entre o exame anatomopatológico e a MMG foi 0,7 e entre o exame anatomopatológico e a US foi 0,7 ( $p<0,05)$. Foram calculados também os coeficientes de correlação de Pearson entre os métodos avaliados, obtendo-se $r=0,7$ entre o EC e a MMG, $r=0,8$ entre o EC e a US e r=0,8 entre a MMG e a US (p<0,05). CONCLUSÕES: o EC, a MMG e a US apresentaram acentuada correlação com a medida anatomopatológica, além de forte correlação entre si, mostrando-se equivalentes como métodos na determinação pré-cirúrgica do tamanho do tumor mamário. No entanto, devido às limitações específicas de cada método, o EC, a MMG e a US devem ser considerados complementares entre si para obtenção de uma medida mais acurada do tumor no câncer de mama.

\section{Abstract}

PURPOSE: to evaluate which method is the best to determine pre-surgically the size of breast cancer: clinical examination, mammography or ultrasonography, using as a reference the anatomopathological exam. METHODS: this study has included 184 patients with palpable-or-not breast lesions, detected by mammography and ultrasonography, that were submitted to surgical resection of the tumor, with histopathological diagnosis of breast cancer. The same examiner evaluated clinically the largest tumoral diameter, through clinical examination, mammography and ultrasonography, and the measurements obtained by each method were correlated with the maximum diameter obtained by the anatomopathological exam. The comparative analysis has been done by Pearson's correlation coefficient ( $r$ ). RESULTS: Pearson's correlation coefficient between the anatomopathological and the clinical exams was 0.8; between the anatomopathological exam and the mammography, 0.7; and between anatomopathological exam and ultrasonography $0.7(p<0.05)$. Pearson's correlation coefficients among the methods evaluated were also calculated and $r=0.7$ was obtained between clinical exam and mammography, $r=0.8$ between clinical examination and utrasonograhy, and $r=0.8$ between mammography and ultrasonography $(p<0.05)$. CONCLUSIONS: clinical examination, mammography and ultrasonography have presented high correlation with the anatomopathological measures, besides high correlations among themselves, what seems to show that they may be used as equivalent methods in the pre-surgical evaluation of the breast tumoral size. Nevertheless, due to specific limitations of each method, clinical examination, mammography and ultrasonography should be seen as complementary to each other, in order to obtain a more accurate measurement of the breast cancer tumor.
Correspondência:

Fernanda Monteiro de Paula Siqueira Rua Esmeralda, 674/ 602 - Bosque da Saúde CEP 78050-050 - Cuiabá/MI Tel: (65) 8115-3100 E-mail: fersiqueira75@yahoo.com.br

Recebido $27 / 11 / 2007$
Trabalho realizado no serviço de Mastologia e Serviço de Diagnóstico por Imagem do Hospital das Clínicas da Faculdade de Medicina da Universidade Federal de Minas Gerais - UFMG - Belo Horizonte (MG), Brasil.

'Pós-graduanda do Departamento de Ginecologia e Obstetrícia da Faculdade de Medicina da Universidade Federal de Minas Gerais - UFMG - Belo Horizonte (MG), Brasil.

2 Professor Adjunto do Departamento de Ginecologia e Obstetrícia da Faculdade de Medicina da Universidade Federal de Minas Gerais - UFMG - Belo Horizonte (MG), Brasil.

${ }^{3}$ Médico Assistente do Serviço de Diagnóstico por Imagem do Hospital das Clínicas da Faculdade de Medicina da Universidade Federal de Minas Gerais - UFMG - Belo Horizonte (MG), Brasil. 


\section{Introdução}

O tamanho tumoral é o fator prognóstico isolado mais importante após o status axilar no câncer de mama, assumindo especial importância nas pacientes com doença localizada e axila clinicamente negativa. A dimensão do tumor tem relação inversa com o prognóstico da paciente com carcinoma mamário, sendo que tumores menores apresentam maior sobrevida e menor índice de recidiva ${ }^{1}$. Além disso, ocorre também uma relação linear entre o tamanho do tumor e a probabilidade de ocorrência de eventuais metástases, sendo que o tempo de desenvolvimento de metástases é menor quanto maior for a dimensão do tumor ${ }^{2}$.

Ainda como fator prognóstico, o tamanho do tumor é essencial para definição do tratamento primário diante do desenvolvimento de técnicas cirúrgicas minimamente invasivas e do incremento da terapia neoadjuvante ${ }^{3}$, para monitoração da resposta ao tratamento quimioterápico ${ }^{4} \mathrm{e}$ para indicação da realização da biópsia do linfonodo sentinela ${ }^{5}$. Portanto, a correta avaliação do tamanho tumoral assume importante papel no planejamento do tratamento do câncer de mama, evitando-se, por um lado, cirurgias mutilantes, com o emprego de terapias neoadjuvantes associadas a cirurgias conservadoras, e, por outro, minimizando os riscos de recidivas pós-operatórias.

A correta determinação do tamanho tumoral é importante não somente para a avaliação individual da paciente com câncer de mama, mas também para a comparação entre os estudos em que o tamanho do tumor é um fator relevante. Até o presente momento, não há consenso na literatura sobre qual o método clínico que melhor avalia o tamanho do tumor no câncer de mama. Diversos estudos fizeram a correlação entre o exame clínico (EC), a mamografia (MMG) e a ultrasonografia (US) com o exame anatomopatológico, porém, os resultados são conflitantes ${ }^{6-22}$. Mais recentemente, a ressonância nuclear magnética (RNM) tem sido estudada para a avaliação do tamanho do tumor e da extensão do câncer de mama. Devido a sua alta sensibilidade, o uso desse método tem crescido como importante complemento da MMG e da US, principalmente no estadiamento inicial da doença ${ }^{23}$. No entanto, por sua baixa especificidade, a RNM pode levar a avaliações falsos-positivas e a superestimativas do tamanho tumoral, limitando seu papel na determinação do tamanho do tumor no câncer de mama ${ }^{24}$. Diante do exposto, a padronização de um ou mais métodos para a determinação do tamanho tumoral torna-se crucial.

O objetivo deste estudo foi avaliar qual o melhor método entre o EC, a MMG e a US na determinação pré-operatória do tamanho tumoral no câncer de mama, tendo como referência o exame anatomopatológico.

\section{Métodos}

Trata-se de um estudo transversal, no qual se comparam os resultados das medidas das lesões mamárias malignas obtidas pelos métodos de EC, MMG e US, tendo como padrão-ouro as medidas do exame anatomopatológico.

Inicialmente, foram selecionadas para o estudo 245 pacientes portadoras de lesões mamárias suspeitas de malignidade admitidas no Serviço de Mastologia do Hospital das Clínicas da Universidade Federal de Minas Gerais (UFMG), no período de agosto de 1997 a agosto de 2002. O EC era realizado com a paciente sentada com os braços soltos e relaxados ao lado do corpo para avaliação das axilas e das fossas supra- e infra-claviculares. Depois, a paciente era posicionada em decúbito dorsal com braço acima da cabeça ou na posição oblíquo-lateral, quando os nódulos localizavam-se nos quadrantes externos, próximos à linha axilar anterior, para espalhar a mama na parede torácica e permitir melhor palpação. $\mathrm{O}$ tumor, então, era fixo entre os dedos do examinador, que o media com uma régua milimetrada flexível. O tamanho considerado era o maior diâmetro encontrado.

Após o EC completo das mamas, a paciente era submetida ao exame ecográfico na mesma posição (decúbito dorsal com braço acima da cabeça ou na posição oblíquo-lateral, quando os nódulos localizavam-se nos quadrantes externos). A avaliação ultra-sonográfica era feita com a utilização do aparelho Siemens Sonoline SL-1, após revisão da MMG realizada previamente. $\mathrm{O}$ contato sonda-pele era feito por um gel apropriado, para melhor definição de imagem e execução do exame. A sonda linear de 7,5 MHz fazia "varredura" de todos os quadrantes da glândula mamária para detectar a lesão suspeita de malignidade, que era explorada em todos os planos (transversal, radial e sagital), sua dimensão era aferida nos três eixos e suas características eram avaliadas para caracterizar o grau de malignidade da imagem observada. $\mathrm{O}$ tamanho considerado era o que apresentava o maior diâmetro aferido, incluindo-se o "halo" hiperecóico, quando existente.

$\mathrm{Na}$ avaliação mamográfica, o tamanho da lesão considerado era o maior diâmetro aferido com régua milimetrada na análise dos filmes em um negatoscópio, nas duas incidências básicas realizadas (crânio-caudal e oblíquo-médiolateral). A medida incluía a ponta mais densa do tumor de um lado ao outro, sem inclusão das espículas muito finas, características das lesões espiculadas. Segundo dados da literatura, a medida mamográfica somente do "núcleo" do tumor, sem a inclusão das espículas, é a que mais se correlaciona com a medida anatomopatológica ${ }^{25}$.

A avaliação clínica, a US e a análise dos filmes mamográficos, com a determinação do maior diâmetro tumoral por cada método, foram realizadas por um mesmo examinador. As medidas obtidas eram armazenadas em um banco de dados informatizado, utilizando-se o programa Epi-Info versão 6.04. Todas as cirurgias foram realizadas 
Tabela 1 - Medidas tumorais obtidas por exame clínico, mamografia, ultra-sonografia e exame anatomopatológico (média, desvio padrão, mediana, mínimo e máximo, n=184)

\begin{tabular}{lccccc}
\hline \multirow{2}{*}{ Métodos } & \multicolumn{3}{c}{ Medidas fumorais (cm) } \\
\cline { 2 - 6 } & Média & Desvio padrão & Mediana & Mínimo & \multicolumn{1}{c}{ Máximo } \\
\hline Exame clínico & 3,6 & 2,4 & 3,0 & 0,0 & 11,0 \\
Mamografia & 2,8 & 1,9 & 2,1 & 0,6 & 10,0 \\
Ulitra-sonografia & 2,6 & 1,4 & 2,2 & 0,7 & 8,0 \\
Anatomopatológico & 3,6 & 2,1 & 3,0 & 0,6 & 13,0 \\
\hline
\end{tabular}

pela equipe de Mastologia do Hospital das Clínicas da UFMG e as medidas anatomopatológicas obtidas por dois patologistas do Serviço de Anatomia Patológica do mesmo hospital. O tamanho do tumor considerado era a medida que apresentava o maior diâmetro aferido com régua milimetrada ao exame macroscópico, ou, nos casos das lesões não vistas ao olho nu, a maior medida obtida pelo exame microscópico da lâmina histológica.

Foram incluídas todas as pacientes que apresentavam lesões mamárias detectadas por MMG e por US, palpáveis ou não, independente do tamanho da lesão, e que foram submetidas à exérese cirúrgica da mesma como tratamento primário, com diagnóstico histopatológico de câncer de mama. Para esses casos selecionados, o tempo decorrido entre a avaliação das pacientes pelos métodos propedêuticos e a exérese cirúrgica para exame anatomopatológico variou de, no mínimo, 20 dias a, no máximo, 90 dias, com média de 28 e mediana de 29 dias. Apenas em dois casos o tempo entre a avaliação clínica inicial e a cirurgia excedeu a 35 dias ( 45 e 90 dias), sendo que, em ambos os casos, o tamanho do tumor não apresentou crescimento nesse período, conforme a reavaliação clínica realizada no dia do procedimento cirúrgico. Foram excluídos 21 casos de diagnóstico histológico de doença benigna mamária, três pacientes encaminhadas para tratamento sistêmico neoadjuvante e 37 pacientes cujas mamografias não estavam disponíveis para análise no Setor de Radiologia do Hospital das Clínicas da UFMG ou que não puderam ser resgatadas de alguma forma. Considerando os critérios de inclusão e exclusão, foi incluído neste estudo um total de 184 pacientes.

Para comparação entre as medidas obtidas por cada método, empregou-se o coeficiente de correlação de Pearson $(r)$. A significância estatística foi considerada com valor de $\mathrm{p}<0,05$.

O estudo foi aprovado pelo Comitê de Ética em Pesquisa (Coep) da UFMG, com o parecer ETIC 339/05.

No grupo das 184 pacientes estudadas, a idade variou entre 30 e 85 anos, com média de $55 \pm 13$ anos e mediana de 53 anos.

O maior diâmetro das lesões, de acordo com o EC, variou de 0 a 11,0 $\mathrm{cm}$, com média de 3,6 $\mathrm{cm}$, mediana de 3,0 cm e desvio padrão de 2,4 cm. Segundo a MMG, o maior diâmetro das lesões variou de 0,6 a 10,0 cm, com uma média de $2,8 \mathrm{~cm}$, mediana de $2,1 \mathrm{~cm}$ e desvio padrão de $1,9 \mathrm{~cm}$. Na avaliação por US, o maior diâmetro das lesões variou de 0,7 a $8,0 \mathrm{~cm}$, com média de $2,6 \mathrm{~cm}$, mediana de $2,2 \mathrm{~cm}$ e desvio padrão de $1,4 \mathrm{~cm}$. Pelo exame anatomopatológico da peça cirúrgica, o maior diâmetro das lesões variou de 0,6 a 13,0 cm, com média de 3,6 cm, mediana de $3,0 \mathrm{~cm}$ e desvio padrão de $2,2 \mathrm{~cm}$ (Tabela 1 ).

Não houve acometimento axilar ao EC em 105 casos $(57,1 \%)$, enquanto $57(31 \%)$ pacientes apresentaram acometimento metastático axilar homolateral sem aderências e 20 (10,9\%), apresentavam gânglios linfáticos homolaterais aderidos ou coalescentes. Duas pacientes que apresentaram recidiva loco-regional foram submetidas a esvaziamento axilar anteriormente à realização dos procedimentos. A mama esquerda apresentou maior acometimento (101 casos; $55 \%$ ) do que a mama direita (83 casos; 45\%), ocorrendo 11 casos de lesões não palpáveis (6\%). Considerando a localização das lesões, a maioria dos casos ocupava o quadrante superior externo (103 casos; $56 \%)$ e seis lesões $(3,3 \%)$ eram multiquadrantes.

A análise histopatológica confirmou a natureza maligna de todas as lesões, diagnosticando 174 casos de carcinomas invasores e dez casos de carcinoma in situ. Entre os carcinomas invasores, 146 casos foram diagnosticados como carcinoma ductal invasor puro; 12 casos de carcinoma lobular invasor; nove casos de carcinoma ductal invasor associado a outros carcinomas; um carcinoma micropapilar; dois casos de carcinoma tubular; dois casos de carcinoma colóide; um carcinoma apócrino e um metaplásico de células fusiformes. Todos os carcinomas não invasores foram diagnosticados como carcinoma ductal in situ.

\section{Resultados}

Calculou-se o coeficiente de correlação de Pearson entre as medidas do maior diâmetro tumoral obtidas pelo exame anatomopatológico e para cada método propedêutico analisado (Tabela 2 e Figuras 1, 2 e 3). $\mathrm{O}$ coeficiente de correlação encontrado entre a medida anatomopatológica e a determinada por EC foi 0,8 . O coeficiente de correlação entre o anatomopatológico e a MMG foi 0,7, enquanto o coeficiente de correlação entre o anatomopatológico e a US foi $0,7(\mathrm{p}<0,05)$.

A análise estatística mostrou que, ao se comparar as medidas anatomopatológicas com as do EC, a diferença 
Tabela 2 - Coeficiente de correlação de Pearson (r) para comparação entre exame anatomopatológico e exame clínico, mamografia e ultra-sonografia ( $n=184)$

\begin{tabular}{lc}
\hline Correlação & $r$ \\
\hline Anatomopatológico versus exame clínico & 0,8 \\
Anatomopatológico versus mamografia & 0,7 \\
Anatomopatológico versus ultra-sonografia & 0,7 \\
\hline
\end{tabular}

Correlação com significância estatística $(p<0,05)$.

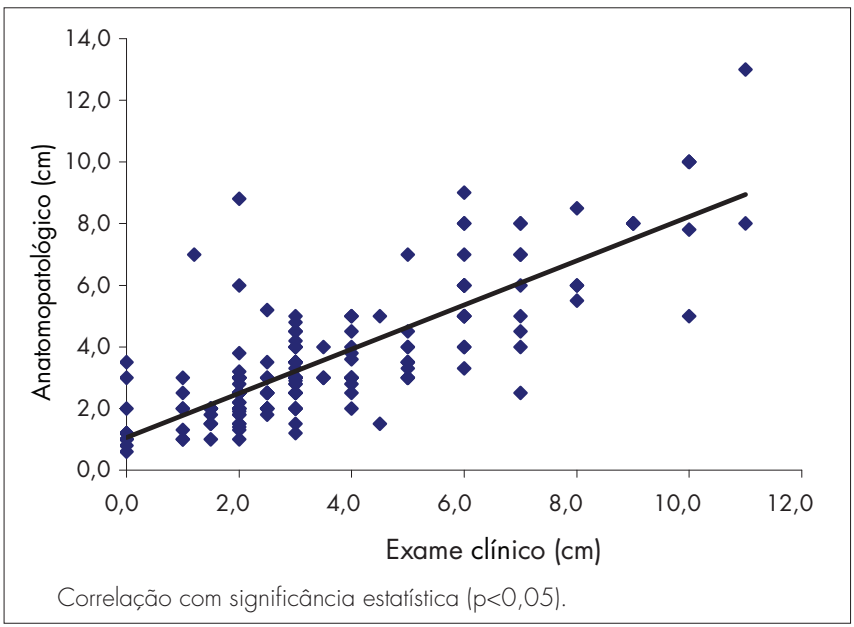

Figura 1 - Correlação entre a medida obtida por anatomopatológico e exame clínico em 184 pacientes.

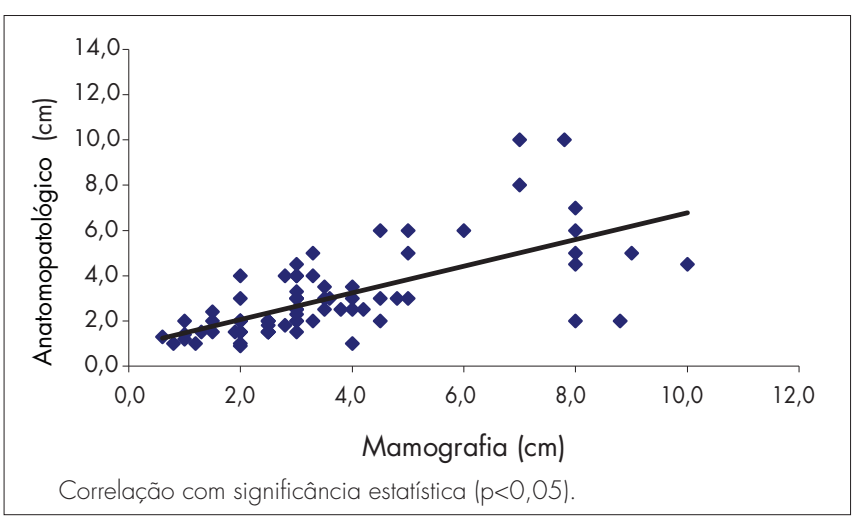

Figura 2 - Correlação entre a medida obtida por anatomopatológico e mamografia em 184 pacientes.

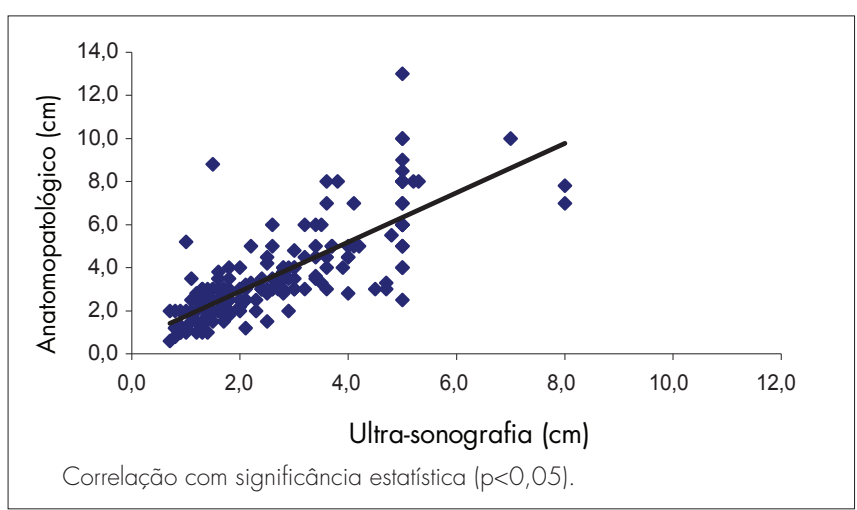

Figura 3 - Correlação entre a medida obtida por anatomopatológico e ultra-sonografia em 184 pacientes. média foi de $0 \pm 1,4 \mathrm{~cm}$, diferença sem significância estatística $(\mathrm{p}=0,70)$. A medida obtida clinicamente foi maior que a medida obtida pelo exame anatomopatológico em 39,1\% dos casos, igual em $16,8 \%$ e menor em $44,1 \%$ das vezes.

Comparando-se as medidas anatomopatológicas com as mamográficas, a análise estatística mostrou uma diferença média de $0,8 \pm 1,4 \mathrm{~cm}$, sendo a diferença significante ( $\mathrm{p}<0,0001)$. A medida mamográfica foi maior que a anatomopatológica em 15,8\% dos tumores, mostrando-se igual também em 15,8\% das vezes. Em 68,4\% dos casos, a MMG obteve uma medida menor que a dimensão anatomopatológica do tumor.

A diferença média entre as medidas do exame anatomopatológico e as da US foi de $1,0 \pm 1,4 \mathrm{~cm}(\mathrm{p}<0,0001)$. $A$ avaliação ecográfica do tamanho tumoral foi maior que a medida anatomopatológica em $11,4 \%$ dos casos; igual em $6 \%$ e menor em $82,6 \%$ dos tumores.

Foram calculados os coeficientes de correlação de Pearson entre os métodos avaliados, obtendo-se $r=0,7$ para a comparação entre EC e MMG, $r=0,8$ entre o EC e a US e $r=0,8$ entre a MMG e a US, sendo todos os valores significantes $(\mathrm{p}<0,005)$. Esses achados demonstram que, além de forte correlação com o exame anatomopatológico, os métodos avaliados apresentam forte correlação entre si na determinação do tamanho tumoral.

\section{Discussão}

A análise dos dados deste estudo demonstrou que os três métodos propedêuticos avaliados apresentaram acentuada correlação com a medida anatomopatológica, mostrando-se equivalentes como métodos na determinação do tamanho tumoral. Esses achados são concordantes com os de outros autores, cujos trabalhos são de metodologia equiparável à nossa ${ }^{6,7,22}$. Um fator importante que deve ser considerado como limitação deste trabalho é a avaliação realizada por um único examinador, que verificava a medida mamográfica do tumor imediatamente antes da execução do EC e da US. O conhecimento prévio da dimensão tumoral pode ter contribuído para elevar a eficácia de cada método realizado subseqüentemente, levando também à acentuada correlação entre eles.

Outros estudos, porém, concluíram que a US seria superior à MMG e ao EC na determinação do tamanho do tumor mamário ${ }^{8-10,19-21}$. A comparação dos resultados desses estudos com os do nosso trabalho é dificultada pelas diversidades quanto à seleção de pacientes, amostragem, prevalência do tamanho da lesão e tipo histológico, parâmetro avaliado como tamanho do tumor, desenho do estudo e diferentes análises estatísticas para comparação entre os métodos. Alguns desses estudos ${ }^{8,11,19-21}$, por exemplo, abordaram preferencialmente lesões menores e mais homogêneas, com expressiva hipoecogenicidade, favorecendo 
a medição por US. No nosso trabalho, 20,6\% dos casos correspondem a lesões maiores do que $5,0 \mathrm{~cm}$, ocorrendo reação desmoplásica com muita frequiência e também bordas imprecisas e espiculadas, o que torna difícil a definição da borda tumoral pelos métodos de imagem.

Em outros trabalhos se observou, também, superioridade da US na determinação do tamanho tumoral em relação à $\mathrm{MMG}$, com boa correlação com a dimensão anatomopatológica, discordando dos nossos achados. No entanto, foram estudos comparativos somente entre os métodos de imagem, não considerando o EC, diferentemente do presente trabalho ${ }^{12-14}$.

Contribuindo para a controvérsia em relação ao melhor método para aferir o tamanho tumoral, outros dois estudos que compararam o EC, a MMG e a US com o exame anatomopatológico em pacientes selecionadas para tratamento sistêmico primário concluíram que a avaliação clínica seria superior às avaliações por métodos de imagem para a determinação do tamanho do tumor residual ${ }^{15,16}$. Diferentemente do nosso trabalho, que avaliou apenas tumores primários em pacientes selecionados para tratamento cirúrgico sem quimioterapia prévia, esses estudos avaliaram tumores avançados, com comparação entre as medidas obtidas após a interferência do tratamento sistêmico sobre o tamanho tumoral. Observou-se, em ambos os estudos, que o EC mostrou-se superior às técnicas de imagem na determinação do tamanho do tumor residual, o que poderia ser explicado pelo fato de que, por um lado, doença residual mínima poderia não ser detectada por esses métodos e, por outro, fibrose e hialinização poderiam ser interpretadas como doença residual na imagem mamográfica e ecográfica.

Os resultados deste trabalho mostraram uma tendência dos métodos de imagem a subestimar o tamanho tumoral de maneira geral, fato confirmado por maior parte dos estudos realizados até o momento ${ }^{6,10-14,17,19-21}$. A MMG pode subestimar o tamanho de uma lesão se o diâmetro máximo do tumor não for captado pelas projeções tradicionais do exame; nos casos de lesões espiculadas e irregulares, com bordas indistintas e pela dificuldade na determinação exata da opacidade da lesão em mamas densas. A subestimativa ecográfica do tamanho tumoral pode ser devida a difícil visibilidade da margem posterior da lesão por inadequada penetração da onda de US e inabilidade de detecção de microcalcificações e de lesões muito pequenas. Por outro lado, a estimativa mamográfica da dimensão do tumor pode ser ampliada devido à variação da distância entre o tumor e o filme, nos casos de incorreto posicionamento da paciente; pela compressão do tumor durante o exame e pela reação desmoplásica tumoral, que pode aumentar a opacidade em torno da lesão. Entretanto, a qualidade do equipamento utilizado para realização dos exames (MMG ou US), a técnica do exame, o correto posicionamento da paciente e a experiência do examinador também contribuem na determinação correta da medida da lesão.

Quanto à determinação clínica do tamanho tumoral, a maioria dos estudos que avalia a tendência de sub- ou superestimativa dos métodos empregados demonstra que o EC tende a superestimar a dimensão da lesão ${ }^{6,8,10,1821}$. São fatores que poderiam explicar essa tendência: a espessura da pele e dos tecidos subcutâneo, adiposo e glandular adjacente ao tumor, edema, obesidade, técnica e instrumento utilizado para aferição, além da experiência do examinador. Em algumas situações, no entanto, o EC também poderia subestimar o tamanho do tumor, como no caso de lesões muito profundas em mamas extremamente volumosas ou no caso de mamas muito densas à palpação, dificultando a distinção entre tumor e tecido glandular normal. Contrariando a maioria dos autores, neste trabalho, entretanto, o EC subestimou $44,1 \%$ das lesões, enquanto superestimou 39,1\% e mostrou-se compatível com o tamanho anatomopatológico em $16,8 \%$ dos casos, achado semelhante ao de outro estudo ${ }^{22}$. Não encontramos explicações para esse fato, mesmo porque a diferença entre as medidas superestimadas e as subestimadas pela avaliação clínica não foi estatisticamente significante.

Relembramos ainda que a correta avaliação do tamanho tumoral assume importante papel no planejamento do tratamento do carcinoma mamário, evitando uma abordagem excessivamente agressiva, com cirurgias mutilantes em um extremo, permitindo, por outro lado, um tratamento mais conservador, com emprego de terapias neoadjuvantes associadas a cirurgias não radicais e minimizando os riscos de recidivas pós-operatórias. Numa época em que cada vez mais procedimentos diagnósticos em mastologia tornam-se disponíveis, entre eles a RNM, demandando aumento de recursos, torna-se essencial a identificação de meios pelos quais os cuidados com a paciente portadora de câncer de mama possam ser mantidos ou incrementados, sem desprezar os custos.

Dessa forma, o EC deve ser valorizado no caso de lesões palpáveis, por se tratar de um exame simples, de rápida realização e praticamente sem custos adicionais. A avaliação clínica parece ser a mais adequada no caso de lesões de grandes dimensões, pois a avaliação pelos métodos de imagem fica prejudicada, uma vez que esses tumores excedem o campo de visão do transdutor e o filme da MMG, ou quando a margem posterior dessas lesões maiores apresenta difícil visibilidade ao US devido à inadequada penetração. Obviamente, o EC apresenta limitações no caso de lesões muito pequenas, que não são palpáveis mesmo por experientes mastologistas, obrigando-nos a recorrer aos métodos de imagem para a determinação do tamanho do tumor. Também nesses casos, devemos considerar, além dos custos, as limitações de cada técnica. Sabendo que a MMG apresenta dificuldade na determinação exata da opacidade da lesão em mamas densas, a US passa a ser o método mais adequado nessa situação. Por outro 
lado, a ecografia tem como maior limitação a inabilidade de detecção de microcalcificações e de lesões muito pequenas, sendo a MMG, então, o método de escolha nesses casos. Quanto ao papel exato da RNM na avaliação do tamanho do tumor de mama, que não foi objeto deste trabalho, é necessária a realização de mais estudos para essa definição. Até o momento, as evidências demonstram a importância desse método como complemento à MMG e US, principalmente na avaliação da extensão da doença.
Os resultados deste estudo demonstram que as medidas tumorais obtidas por EC, MMG e US apresentam forte correlação com a obtida por exame anatomopatológico, além de forte correlação entre si, sendo, portanto, equivalentes na determinação do tamanho do tumor mamário. Concluímos, porém, que devido às limitações específicas de cada método, o EC, a MMG e a US devem ser considerados complementares entre si para obtenção de uma medida mais acurada do tumor no câncer de mama.

\section{Referências}

1. Yeatman TJ, Bland KI. Staging of breast cancer. In: Bland KI, Copeland EM, editors. The breast. Philadelphia: WB Saunders; 1991. p. 313-30.

2. Harris J, Morrow M, Norton L. Malignant tumors of the breast. In: DeVita VT Jr, Hellman S, Rosenberg SA, editors. Cancer: principles \& practice of Oncology. 5th ed. Philadelphia: Lippincott-Raven; 1997. p. 1541-616.

3. Veronesi U, Luini A, Galimberti V, Zurrida S. Conservation approaches for the management of stage I/II carcinoma of the breast: Milan Cancer Institute trials. World J Surg. 1994; 18(1):70-5.

4. Yeh E, Slanetz P, Kopans DB, Rafferty E, Georgian-Smith D, Moy L, et al. Prospective comparison of mammography, sonography, and MRI in patients undergoing neoadjuvant chemotherapy for palpable breast cancer. AJR Am J Roentgenol. 2005;184(3):868-77.

5. Zurrida S, Galimbert V, Leuini A. A dissecção axilar no carcinoma de mama. In: Veronesi U, editor. Mastologia oncológica. Rio de Janeiro: MEDSI; 2002. p. 302-11.

6. Pain JA, Ebbs SR, Herm RP, Lowe S, Bradbeer JW. Assessment of breast cancer size: a comparison of methods. Eur J Surg Oncol. $1992 ; 18(1): 44-8$.

7. Bui KL, Lamb TJ, Rapelyea JA, Schoonjans JM, Brem RF. Accuracy of mammographic and sonographic assessment of preoperative breast cancer size. J Women's Imaging. 2003;5(3):128-33.

8. Fornage BD, Toubas $O$, Morel M. Clinical, mammographic and sonographic determination of preoperative breast cancer size. Cancer. 1987;60(4):765-71.

9. Serrano Migallón JA, Sandoval Guerrero FJ, Miranda Hernández H, Martinez Macías R. Estudio comparativo entre clínica, mastografía y ultrasonido, para determinar el tamaño de las lesiones mamarias. Rev Inst Nac Cancerol (Mex). 1993;39(4):1931-6.

10. Meden H, Neues KP, Röben-Kämpken S, Kuhn W. A clinical, mammographic, sonographic and histologic evaluation of breast cancer. Int J Gynaecol Obstet. 1995;48(2):193-9.

1 1. Madjar H, Ladner HA, Sauerbrei W, Oberstein A, Prömpeler H, Pfleiderer A. Preoperative staging of breast cancer by palpation, mammography and high-resolution ultrasound. Ultrasound Obstet Gynecol. 1993;3(3): 185-90.

12. Tresserra F, Feu J, Grases PJ, Navarro B, Alegret X, Férnandez-Cid A. Assessment of breast cancer size: sonographic and pathologic correlation. J Clin Ultrasound. 1999;27(9):485-91.

13. Skaane $P$, Skjørten F. Ultrasonographic evaluation of invasive lobular carcinoma. Acta Radiol. 1999;40(4):369-75.

14. Hieken TJ, Harrison J, Herreros J, Velasco JM. Correlating sonography, mammography and pathology in the assessment of breast cancer size. Am J Surg. 2001;182(4):351-4.
15. Herrada J, lyer RB, Atkinson EN, Sneige N, Budzdar AU, Hortobagyi GN. Relative value of physical examination, mammography and breast sonography in evaluating the size of primary tumor and regional lymph node metastasis in women receiving neoadjuvant chemotherapy of locally advanced breast carcinoma. Clin Cancer Res. 1997;3(9): 1565-9.

16. Fiorentino C, Berruti A, Bottini A, Bodini M, Brizzi MP, Brunelli A, et al. Accuracy of mammography and echography versus clinical palpation in the assessment of response to primary chemotherapy in breast cancer patients with operable disease. Breast Cancer Res. 2001;69(2):143-51.

17. Finlayson CA, MacDermott TA. Ultrasound can estimate the pathologic size of infiltrating ductal carcinoma. Arch Surg. 2000; 135(2): 158-9.

18. Forouhi P, Walsh JS, Anderson TJ, Chetty U. Ultrasonography as a method of measuring breast tumour size and monitoring response to primary systemic treatment. Br J Surg. 1994;81 (2):223-5.

19. Pierie JPEN, Perre Cl, Levert LM, de Hooge P. Clinical assessment, mammography and ultrasonography as methods of measuring the size of breast cancer: a comparision. Breast. $1998 ; 7(5): 247-50$.

20. Bosch AM, Kessels AG, Beets GL, Rupa JD, Koster D, van Engelshoven JM, et al. Preoperative estimation of the pathological breast tumour size by physical examination, mammography and ultrasound: a prospective study on 105 invasive tumours. Eur J Radiol. 2003;48(3):285-92.

21. Shoma A, Moutamed A, Ameen M, Abdelwahab A. Ultrasound for accurate measurement of invasive breast cancer tumor size. Breast J. 2006;12(3):252-6.

22. Heusinger K, Löhberg C, Lux MP, Papadopoulos T, Imhoff K, Schulz-Wendtland $R$, et al. Assessment of breast cancer tumor size depends on method, histopathology and tumor size itself. Breast Cancer Res Treat. 2005;94(1):17-23.

23. Van Goethem M, Tjalma W, Schelfout K, Verslegers I, Biltjes I, Parizel P. Magnetic resonance imaging in breast cancer. Eur J Surg Oncol. 2006;32(9):901-10.

24. Van Goethem M, Schelfout K, Dijckmans L, Van Der Auwera JC, Weyler J, Verslegers I, et al. MR mammography in the preoperative staging of breast cancer in patients with dense breast tissue: comparison with mammography and ultrasound. Eur Radiol. 2004;14(5):809-16.

25. Flanagan FL, McDermott MB, Barton PT, Pilgram TK, Dehdashti F, Wick MR, et al. Invasive breast cancer: mammographic measurement. Radiology. 1996;199(3):819-23. 